\title{
ROCK1, a novel target of miR-145, promotes glioma cell invasion
}

\author{
XIN WAN, QUAN CHENG, RENJUN PENG, ZHIMING MA, ZIGUI CHEN, \\ YIQIANG CAO and BING JIANG
}

Department of Neurosurgery, Xiangya Hospital, Central South University, Changsha, Hunan 410008, P.R. China

Received September 3, 2013; Accepted February 7, 2014

DOI: $10.3892 / \mathrm{mmr} .2014 .1982$

\begin{abstract}
Malignant glioma is the most common type of cancer in the central nervous system, with highly invasive characteristics. The Rho-associated protein kinase (ROCK1) has been found to act as key regulator of actin cytoskeleton reorganization, a process closely associated with cancer cell invasion. microRNA-145 (miRNA-145) has been recently shown to act as a suppressor in several types of tumor, including glioma. However, the exact regulatory mechanism by which miR-145 inhibits glioma still remains to be uncovered. In this study, we report that the miR-145 level was significantly reduced in glioma tissues and in the human glioma cell lines U87 and U251, as compared to matched adjacent and normal brain tissues. We then identified the ROCK1 gene as a novel target of miR-145. The expression of ROCK1 was markedly upregulated in glioma tissues, as well as in U87 and U251 cells. Moreover, miR-145 significantly inhibited ROCK1 protein expression in U87 cells. We further show that miR-145 transfection considerably reduced the invasive ability of U87 cells, and was accompanied by the downregulation of matrix metalloproteinase 2 and 9, an effect which could be attenuated by overexpression of ROCK1. In conclusion, the present study suggests that miR-145 can inhibit U87 glioma cell invasion, at least partially via downregulation of the RhoA/ROCK1 pathway. In conclusion, this is the first study to report that ROCK1, as a novel target of miR-145, acts as a positive regulator of glioma cell invasion. Therefore, ROCK1 may constitute a promising target for glioma treatment.
\end{abstract}

\section{Introduction}

Malignant glioma is the most common type of cancer in the central nervous system, with highly invasive characteristics (1). Research on malignant glioma invasion has recently

Correspondence to: Professor Bing Jiang, Department of Neurosurgery, Xiangya Hospital, Central South University, Xiangya Road 87, Changsha, Hunan 410078, P.R. China

E-mail: csubingjiang@163.com

Key words: glioma, invasion, microRNA-145, ROCK1, target gained considerable interest; however, the exact molecular mechanisms underlying malignant glioma invasion remain unclear, mainly since invasion involves a variety of complex processes, as well as regulatory mechanisms $(2,3)$.

microRNAs (miRNAs) are a type of endogenous non-coding RNA. They can regulate gene expression through binding to the 3'-untranslated region (3'-UTR) of target mRNAs, resulting in either mRNA degradation or translational repression (4). Therefore, miRNAs generally act as endogenous agents of RNA interference. Accumulating evidence has shown that dysregulation of the expression of some miRNAs plays an essential role in the development and progression of various cancers (5), which suggests that these miRNAs have oncogenic or anti-oncogenic functions. Moreover, several miRNAs have been shown to be involved in brain physiology and tumorigenesis, including glioma $(6,7)$. miR-145 has been reported to function as a tumor-suppressive RNA in glioma, and several gene targets of miR-145 playing a role in gliomas have been identified, including Sox9, adducin 3 (ADD3), ADAM17, and NEDD9 (8-11). However, the mechanism by which miR-145 regulates glioma has still not been fully uncovered.

The reorganization of the actin cytoskeleton leads to cellular morphological changes, as well as alterations in cellular functions, including proliferation, adhesion and migration $(12,13)$. Rho family proteins interact with the actin cytoskeleton, and are hence involved in the regulation of the cytoskeleton reorganization (14). As a downstream effector of Rho, the Rho-associated protein kinase (ROCK1) is a serine-threonine protein kinase. It was shown that when ROCK1 binds to the active GTP-bound form of Rho, it is activated and then interacts with the actin cytoskeleton to promote the formation of stress fibers, as well as focal adhesion. Moreover, ROCK1 has been reported to provide a feedback mechanism and regulate the activity of upstream proteins Racl and RhoA, which act as key regulators in the reorganization of the actin cytoskeleton (15). Since the reorganization of the actin cytoskeleton plays a role in cancer cell migration and invasion, ROCK is believed to be involved in the process of cancer invasion and metastasis. In fact, ROCK1 has been reported to associate with several types of malignant tumors, including osteosarcoma, prostate carcinoma, and bladder cancer (16-18). However, its exact role in glioma remains unknown. 
The present study identified, for the first time to the best of our knowledge, the ROCK1 gene as a direct target of miR-145, and showed that this microRNA can inhibit U87 glioma cell invasion, at least partially via downregulating the RhoA/ROCK1 pathway.

\section{Materials and methods}

Reagents and materials. Dulbecco's modified Eagle's medium (DMEM), fetal bovine serum (FBS), TRIzol, Lipofectamine 2000, TaqMan microRNA assays, Platinum ${ }^{\circledR}$ RTS SYBR ${ }^{\circledR}$ Green qPCR SuperMix-UDG and miR-145 mimics were purchased from Life Technologies (Carlsbad, CA, USA). The QuikChange Site-Directed Mutagenesis kit was purchased from Stratagene (La Jolla, CA, USA). The PGL3 Luciferase Reporter vector and the Dual Luciferase Assay kit were purchased from Promega (Madison, WI, USA). Mouse monoclonal antibodies anti-ROCK1 and anti-GAPDH, and rabbit anti-mouse secondary antibody were purchased from Santa Cruz Biotechnology, Inc. (Santa Cruz, CA, USA). PcDNA3.1 $1^{+}$-ROCK1 plasmid was constructed by Sup Biology (Changsha, China). A 24-well Transwell chamber, pre-coated with dried extracellular matrix was obtained from Corning Inc. (Corning, NY, USA). ELISA kits for matrix metalloproteinase (MMP) 2 and 9 were purchased from R\&D Systems, Inc. (Minneapolis, MN, USA).

Collection of tissue specimens. Written informed consent was obtained from all patients in this study, which was approved by the Ethical Committee of the First Xiangya Hospital of Central South University (Changsha, China). Twenty glioma tissues and their matched adjacent tissues, as well as eight normal brain tissues were obtained from patients at the Department of Neurosurgery, First Xiangya Hospital of Central South University, from March to June 2012. Before surgery, no patient had undergone hormone, radio-, or chemotherapy. All samples were immediately snap-frozen in liquid nitrogen after surgical removal, and stored at $-80^{\circ} \mathrm{C}$ until use.

Cell culture. Human glioma cell lines U251 and U87 were purchased from the Cell Bank of the Chinese Academy of Science and cultured in DMEM supplemented with 10\% FBS and $1 \%$ penicillin/streptomycin at $37^{\circ} \mathrm{C}$ with $5 \% \mathrm{CO}_{2}$.

Transfection. Lipofectamine 2000 was used in the transfection experiments in accordance to the manufacturer's instructions. Briefly, $10^{5}$ cells were harvested, resuspended, seeded in a 6-well plate and cultured at $37^{\circ} \mathrm{C}, 5 \% \mathrm{CO}_{2}$ for $24 \mathrm{~h}$. The U87 cells were transfected with $200 \mathrm{nM}$ miR-145 mimic or negative control (NC) miRNA mimic, or $4 \mu \mathrm{g}$ of the pcDNA3.1 $1^{+}$-ROCK1 plasmid.

$R N A$ extraction and quantitative reverse transcription-polymerase chain reaction ( $q R T-P C R)$. The TRIzol agent was used to extract total RNA from tissues and cells. To detect mature miR-145 expression, the TaqMan MicroRNA Assay kit was used in accordance with the manufacturer's instructions. All reactions were run in a 7500 Fast Real-Time PCR system (Life Technologies, Waltham, MA, USA). Independent experiments were repeated three times for each sample and the relative expression levels of genes were assessed by using a comparative $\mathrm{Ct}$ method. For mRNA quantification through qRT-PCR analysis, we followed the manufacturer's protocol and used Platinum ${ }^{\circledR}$ RTS SYBR $^{\circledR}$ Green qPCR SuperMix-UDG and the following specific primers: ROCK1, 5'-GGTGGTCGGTTGGGGTATTTT-3' (forward) and 5'-CGCCCTAACCTCACTTCCC-3' (reverse); glyceraldehyde phosphate dehydrogenase (GAPDH), 5'-ACAACTTTGGTATCGTGGAAGG-3' (forward) and 5'-GCCATCACGCCACAGTTTC-3' (reverse).

Luciferase activity assay. To determine whether ROCK1 is a direct target of miR-145, the pGL3 Luciferase Reporter vector was used. The putative target sequences on the 3'-UTR of ROCK1 were analyzed by TargetScan (19). The primer sequences for the wild-type 3'-UTR of ROCK1 were: 5'-CGCG GCCGCTAGTCTGTGGAATCGTGTGGGAT-3' (forward) and 5'-CTAGATCCCACACGATTCCACAGACTAGCG GCCGCGAGCT-3' (reverse). The QuikChange Site-Directed Mutagenesis kit was used based on the manufacturer's protocols to construct the mutant 3'-UTR of ROCK1, bearing a three-nucleotide substitution (UGG to CAA) within the miR-145 target sequence (Fig. 2A).

For the luciferase activity assay, we co-transfected U87 and U251 cells with miR-145 mimic and the pGL3 Luciferase Reporter vector bearing either the wild-type or the mutant type 3'-UTR of ROCK1. Luciferase activities were determined by the Dual Luciferase assay $24 \mathrm{~h}$ after transfection according to the manufacturer's instructions. All experiments were performed in triplicate.

Invasion assay. Cells were harvested and resuspended in DMEM without FBS. For the invasion assay, $2 \times 10^{5}$ cells were added into the upper Transwell chamber, where DMEM without FBS was added. DMEM containing 10\% FBS was added to the lower chamber. After incubation for $6 \mathrm{~h}$, cells were fixed with $3.7 \%$ formaldehyde and stained with crystal violet staining solution. The cells that had not passed through the $8.0-\mu \mathrm{m}$ pore polycarbonate membrane between the chambers were removed. Five fields of the lower surface of the membrane were randomly selected to determine the number of cells that passed through the membrane.

Western blotting. Glioma tissues or U87 cells were solubilized in cold radioimmunoprecipitation assay lysis buffer. Then, proteins (15 $\mu \mathrm{g}$ per lane) were separated with $10 \%$ SDS-PAGE, and transferred to a polyvinylidene difluoride membrane. Membranes were then blocked in 5\% non-fat dried milk in phosphate-buffered saline solution with Tween 20 (PBST) for $4 \mathrm{~h}$ and then incubated for $3 \mathrm{~h}$ with mouse anti-ROCK1 monoclonal antibody (1:400), or mouse anti-GAPDH monoclonal antibody (1:200). After incubation with rabbit anti-mouse secondary antibody $(1: 40,000)$ for 40 min, enhanced chemiluminescence reagent was used to detect the signals on the membranes. We then used the Image-Pro ${ }^{\circledR}$ Plus 6.0 software (Media Cybernetics, Rockville, MD, USA) to perform gray scale scanning, and calculate the relative values of protein expression.

Statistical analysis. Statistical analyses were performed with the SPSS 17.0 software (IBM, Armonk, NY, USA). Quantified 
A

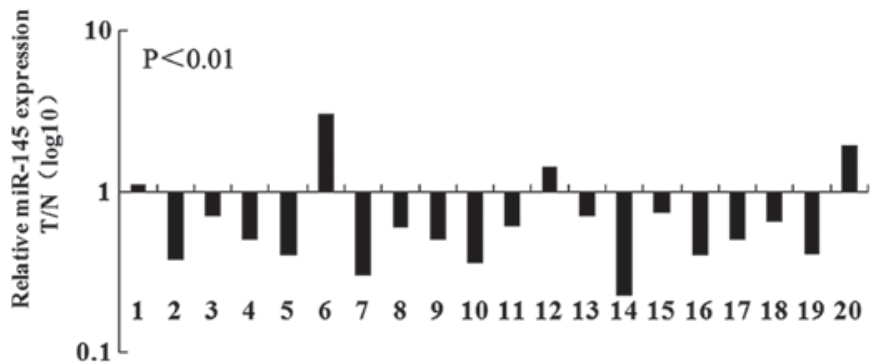

B

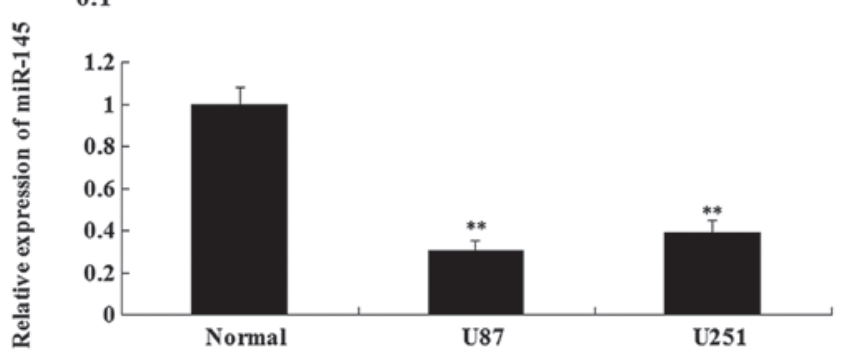

Figure 1. Quantitative reverse transcription-polymerase chain reaction (qRT-PCR) was performed to determine the relative expression of microRNA-145 (miR-145) in (A) 20 samples of glioma and their matched adjacent tissues and (B) in healthy brain tissues and in the glioma cell lines U87 and U251. U6 was used as an endogenous control in all experiments. T/N, tumor/normal; normal, healthy brain tissues.*** $\mathrm{P}<0.01$ vs. normal.

A

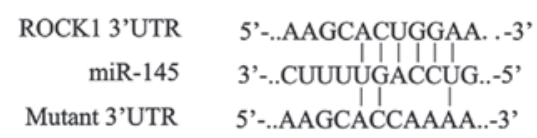

Mutant 3'UTR 5 '...AAGCACCAAAA...-3'

$\mathbf{B}$



C
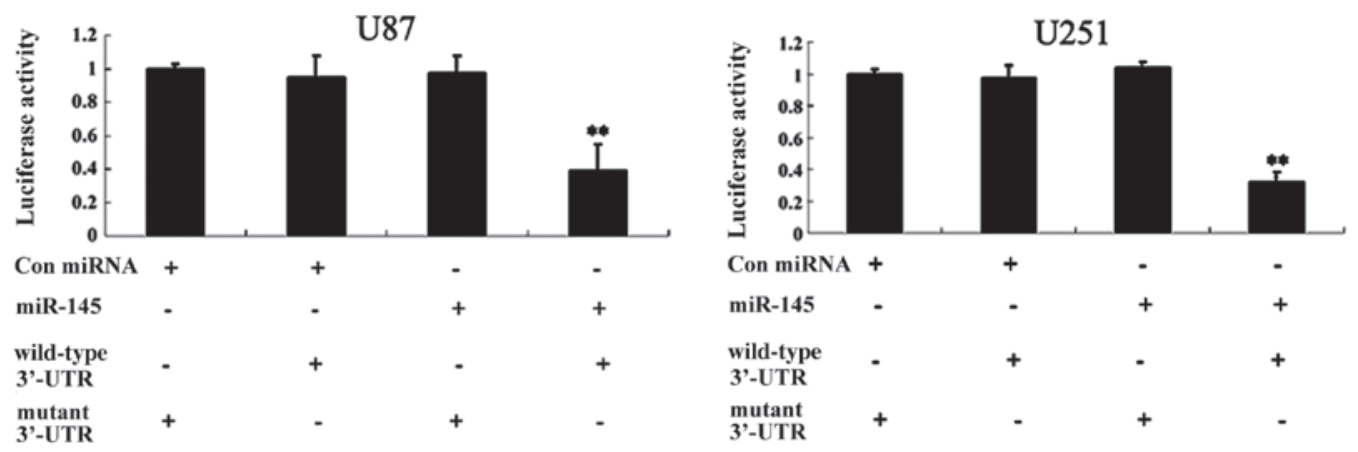

Figure 2. (A) Illustration of the putative target sites of microRNA-145 (miR-145) in the 3'-untranslated region (3'-UTR) of the Rho-associated protein kinase gene (ROCK1) according to the TargetScan prediction. The mutant ROCK1 3'-UTR sequence was constructed to bear a substitution of UGG to CAA within the miR-145 target sequence based on this prediction. (B) Western blotting was performed to determine the protein level of ROCK1 in U87 and U251 cells after transfection with negative control (NC) miRNA or miR-145, respectively. The glyceraldehyde phosphate dehydrogenase (GAPDH) protein was used as an endogenous control. (C) A luciferase activity assay was performed to study the effect of miR-145 transfection on luciferase activity of the reporter vector bearing wild-type or mutant ROCK1 3'-UTR in U87 and U251 cells. Con miR, control miRNA. ${ }^{* *} \mathrm{P}<0.01$ vs. control group.

data are expressed as mean of at least triplicate samples \pm standard deviation. One-way analysis of variance or Student's t-tests were applied to assess the statistical significance of observed differences. $\mathrm{P}<0.05$ was considered to indicate a statistically significant difference.

\section{Results}

miR-145 expression is significantly reduced in glioma tissues and U87 and U251 cells. To investigate the biological signifi- cance of miR-145, we first applied qRT-PCR to determine its expression in 20 glioma and their matched adjacent tissue samples, and found that the miR-145 level is significantly reduced in glioma tissues, compared to the matched adjacent tissues (Fig. 1A). Next, the expression level of miR-145 was examined in the glioma cell lines U87 and U251, as well as in normal brain tissues. As demonstrated in Fig. 1B, the expression of miR-145 was markedly reduced in U87 and U251 cells compared to normal brain tissues. These findings suggest that miR-145 might act as a tumor suppressor in malignant glioma. 


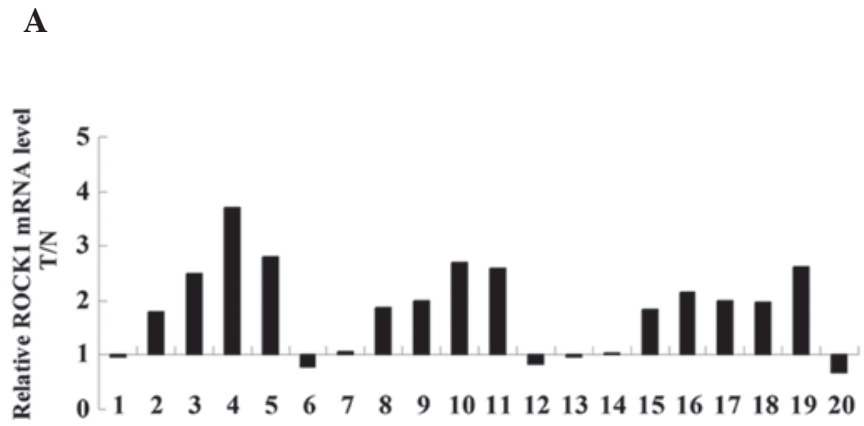

B

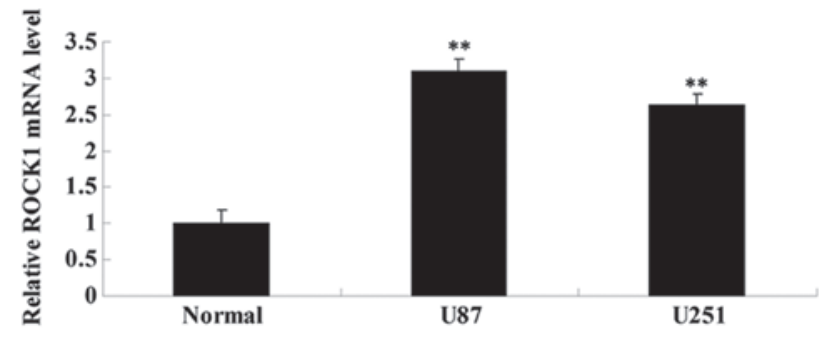

C

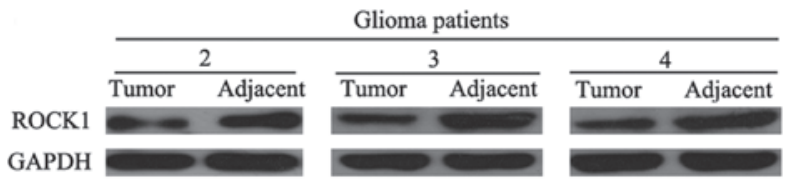

D

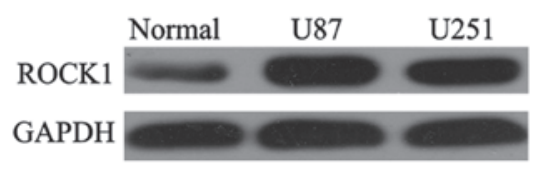

Figure 3. Quantitative reverse transcription-polymerase chain reaction (qRT-PCR) was performed to determine the relative mRNA expression of Rho-associated protein kinase gene (ROCK1) in (A) 20 glioma nd their matched adjacent tissue samples and (B) in three healthy brain tissues and the glioma cell lines U87 and U251. U6 was used as an endogenous control in all experiments. Normal, healthy brain tissues. ${ }^{* *} \mathrm{P}<0.01$ vs. normal. Western blotting was performed to determine the protein level of ROCK1 in (C) 20 glioma and their matched adjacent tissue samples (three representative blots are shown) and (D) in healthy brain tissues and the glioma cell lines U87 and U251. The glyceraldehyde phosphate dehydrogenase (GAPDH) protein was used as an endogenous control in all experiments. $\mathrm{T} / \mathrm{N}$, tumor/normal.

miR-145 directly targets ROCK1 in U87 and U251 cells. To further investigate the regulatory role of miR-145 in glioma, we applied bioinformatic analysis to predict its targets, and the TargetScan software analysis identified the gene ROCK1 as a putative target of miR-145 (Fig. 2A). The ROCK1 protein has been suggested to play a role in cancer cell migration, and the combination of ROCK1 inhibition and an anti-neoplastic agent has been recommended for glioma treatment $(20,21)$. Thus, we hypothesized that miR-145 may be involved in the regulation of glioma cell invasion by directly targeting ROCK1. To verify this hypothesis, we transfected U87 and U251 cells with miR-145 mimic, and determined the expression level of ROCK1 by western blotting. As shown in Fig. 2B, miR-145

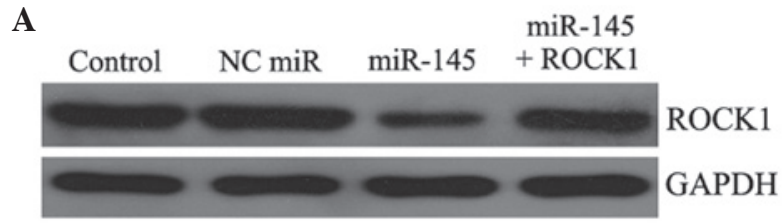

B
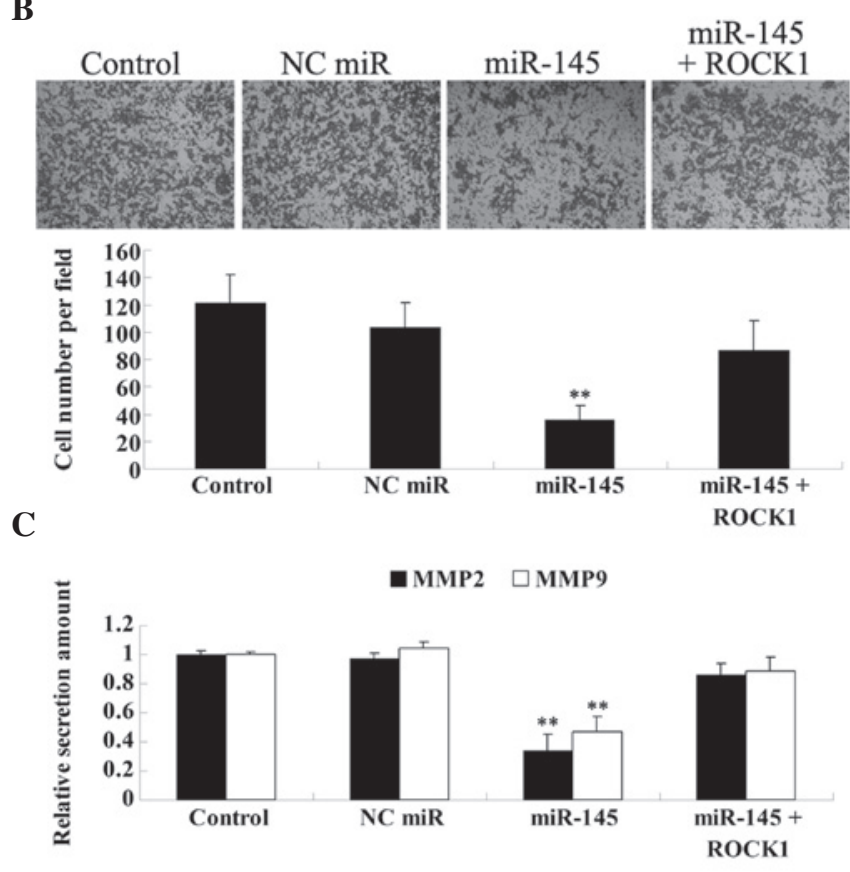

D

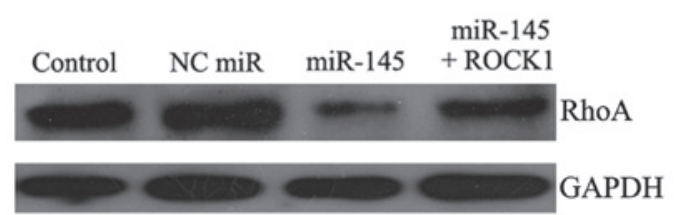

Figure 4. (A) Western blot analysis was performed to determine the protein level of the Rho-associated protein kinase gene (ROCK1) in U87 cells. The glyceraldehyde phosphate dehydrogenase (GAPDH) protein was used as an endogenous control. (B) An invasion assay was performed to investigate the effects of microRNA-145 (miR-145) and ROCK1 on U87 cell invasion. Five fields were randomly chosen, and cell number per field was counted. (C) An ELISA assay was performed to determine the relative secretion of matrix metalloproteinase (MMP) 2 and 9 in U87 cells. (D) Western blot was used to examine the protein level of RhoA in U87 cells. GAPDH was used as an endogenous control. Control, non-transfected U87 cells; NC miR, U87 cells transfected with the negative control miRNA; miR-145 + ROCK1: U87 cells co-transfected with miR-145 mimic and the pcDNA3.1 $1^{+}$-ROCK1 plasmid. ${ }^{* *} \mathrm{P}<0.01$ vs. control.

significantly reduced the ROCK1 protein level compared to the control group (non-transfected cells), while transfection with the NC miRNA had no effect. To further investigate whether a direct interaction exists between miR-145 and ROCK1, the luciferase activity assay was performed. As demonstrated in Fig. 2C, co-transfection of U87 and U251 cells with miR-145 along with the wild-type 3'-UTR of ROCK1 caused a notable decrease in luciferase activity; while this activity was not affected by co-transfection with miR-145 and the mutant 3'-UTR of ROCK1. Based on these findings, we conclude that ROCK1 is a direct target of miR-145, and miR-145 can inhibit ROCK1 protein expression. 
The expression of ROCK1 is markedly increased in glioma tissue and in U87 and U251 cells. We further performed qRT-PCR and western blotting assays to determine the mRNA and protein levels of ROCK1 in glioma tissues, their matched adjacent tissues, as well as in U87 and U251 cells. As demonstrated in Fig. 3A, the ROCK1 mRNA level in glioma tissues was higher than that observed in their matched adjacent tissues. In addition, ROCK1 mRNA expression was upregulated in U87 and U251 cells compared to normal brain tissues (Fig. 3B). The qRT-PCR results were confirmed by western blotting analysis, which demonstrated that ROCK1 is also upregulated at the protein level in glioma compared to matched adjacent tissues, as well as in U87 and U251 cells compared to normal brain tissues (Fig. 3C and D).

Overexpression of ROCK1 attenuates the suppressive effect of miR-145 on U87 cell invasion. To verify our hypothesis that miR-145 inhibits the invasive ability of U87 cells in a ROCK1-dependent manner, we transfected U87 cells with miR-145 mimic and the pcDNA3.1 $1^{+}$ROCK1 plasmid. The effect of transfection was confirmed by western blotting (Fig. 4A). The invasion assay showed that miR-145 significantly reduces the invasive ability of U87 cells, while ROCK1 overexpression partially, but not significantly, rescues this phenotype (Fig. 4B).

It has been well established that MMP2 and MMP9, secreted by cancer cells, play crucial roles in cancer invasion and metastasis (22). Thus, we used an ELISA assay to examine the protein levels of the two proteins in the culture medium. As shown in Fig. 4C, the protein levels of MMP2 and MMP9 were markedly and significantly reduced in U87 cells transfected with miR-145 mimic, as compared with the control group. ROCK1 overexpression also attenuated the inhibitory effect of miR-145 on MMP2 and MMP9 secretion, albeit again not in a significant manner.

To further investigate the molecular mechanism underlying the miR-145/ROCK1-mediated reduction in U87 cell invasion, we focused on the Rho/ROCK pathway, which has been found to associate with cancer metastasis by regulating the actin cytoskeleton reorganization. As shown in Fig. 4D, enhanced expression of miR-145 notably reduced the protein expression of RhoA in U87 cells, while ROCK1 overexpression attenuated this effect.

\section{Discussion}

Accumulating evidence has recently revealed the crucial suppressive role of miR-145 in various types of cancer, including bladder, urothelial, breast, prostate and colon carcinoma (23-25). In this study, we found that in glioma tissues classified as high grade based on World Health Organization (WHO) standards, as well as in the glioblastoma cell lines U87 and U251, the expression level of miR-145 is significantly reduced compared to normal brain tissues. Our findings are consistent with other studies $(8,26)$. For instance, Lee et al showed that miR-145 is downregulated in gliomas, and its low expression in glioblastomas predicts poor prognosis (6). Based on these and our findings, we suggest that miR-145 may play an essential role in glioma progression.

Recently, Rani and colleagues reported that miR-145 inhibits the proliferation, adhesion and invasion of glioblastoma cells by targeting the oncogenic proteins Sox 9 and ADD3 (8). Lee et al showed that miR-145 regulates glioma cell migration by targeting CTGF, which further reduces the expression of SPARC, an important extracellular protein involved in cancer cell migration (26). In addition, NEDD9 and ADAM17 were also recently identified as targets of miR-145, and might become important biomarkers of glioma progression (11). Despite these findings, the detailed role of miR-145 in glioma progression still needs to be fully investigated.

Since miR-145 has been shown to act as a suppressor in glioma, mainly via inhibiting the protein expression of its targets, we hypothesized that a few of its targets that participate in miR-145-dependent regulation of biological processes in glioma cells might not have been identified yet. As ROCK1 has been suggested to associate with several types of cancer through its regulatory role in cytoskeleton reorganization (27), we investigated whether ROCK1 is a miR-145 target in U87 cells, as suggested the bioinformatic prediction based on the 3'-UTR sequence of this gene. Western blot analysis, as well as the luciferase reporter assay data, confirmed that ROCK1 is a novel, direct target of miR-145. We further showed that ROCK1 is upregulated in malignant glioma tissues and in the U87 and U251 cell lines, while transfection with miR-145 led to a decrease in its protein level in U87 cells. These findings indicate that ROCK1, directly regulated by miR-145, may be associated with glioma progression.

As the Rho/ROCK pathway has been found to be involved in the progression and metastasis of several types of cancer $(28,29)$, we further investigated its implication and showed that miR-145 has a suppressive effect on U87 cell invasion, at least partially by downregulating the RhoA/ROCK1 pathway. Notably, we found that miR-145 not only inhibits ROCK1 gene and protein expression, but also downregulates the expression of the upstream small GTPase RhoA at the protein level, indicating that ROCK1 may have diverse effects on the functional balance of small GTPases. Tang et al recently also proposed a feedback regulation mechanism for ROCK1 and RhoA (30). Moreover, the specific ROCK inhibitor Y-27632 was shown to inhibit tumor growth and metastasis (31), which renders this inhibitor a promising agent for the targeted therapy of glioma.

In conclusion, the present study provided evidence, for the first time to the best of our knowledge, that miR-145 can inhibit invasion of glioma cells, at least partially through its inhibitory effect on the Rho/ROCK1 pathway. More importantly, since some compounds have been reported to act as ROCK1 inhibitors, ROCK1 may constitute the new molecular target for the treatment of glioma.

\section{References}

1. Wang $\mathrm{Y}$ and Jiang $\mathrm{T}$ : Understanding high grade glioma: molecular mechanism, therapy and comprehensive management. Cancer Lett 331: 139-146, 2013.

2. Nakada M, Kita D, Teng L, et al: Receptor tyrosine kinases: principles and functions in glioma invasion. Adv Exp Med Biol 986: 143-170, 2013.

3. Kwiatkowska A and Symons M: Signaling determinants of glioma cell invasion. Adv Exp Med Biol 986: 121-141, 2013.

4. Nagadia R, Pandit P, Coman WB, Cooper-White J and Punyadeera $\mathrm{C}$ : miRNAs in head and neck cancer revisited. Cell Oncol (Dordr) 36: 1-7, 2013. 
5. Baer C, Claus R and Plass C: Genome-wide epigenetic regulation of miRNAs in cancer. Cancer Res 73: 473-477, 2013.

6. Wang Q, Li P, Li A, et al: Plasma specific miRNAs as predictive biomarkers for diagnosis and prognosis of glioma. J Exp Clin Cancer Res 31: 97, 2012.

7. Asadi-Moghaddam K, Chiocca EA and Lawler SE: Potential role of miRNAs and their inhibitors in glioma treatment. Expert Rev Anticancer Ther 10: 1753-1762, 2010.

8. Rani SB, Rathod SS, Karthik S, Kaur N, Muzumdar D and Shiras AS: MiR-145 functions as a tumor-suppressive RNA by targeting Sox 9 and adducin 3 in human glioma cells. Neuro Oncol 15: 1302-1316, 2013.

9. Lu Y, Chopp M, Zheng X, Katakowski M, Buller B and Jiang F: MiR-145 reduces ADAM17 expression and inhibits in vitro migration and invasion of glioma cells. Oncol Rep 29: 67-72, 2013

10. Lee SJ, Kim SJ, Seo HH, et al: Over-expression of miR-145 enhances the effectiveness of HSVtk gene therapy for malignant glioma. Cancer Lett 320: 72-80, 2012

11. Speranza MC, Frattini V, Pisati F, et al: NEDD9, a novel target of miR-145, increases the invasiveness of glioblastoma. Oncotarget 3: 723-734, 2012.

12. Chen RH, Wang WJ and Kuo JC: The tumor suppressor DAP-kinase links cell adhesion and cytoskeleton reorganization to cell death regulation. J Biomed Sci 13: 193-199, 2006.

13. Becart S and Altman A: SWAP-70-like adapter of T cells: a novel Lck-regulated guanine nucleotide exchange factor coordinating actin cytoskeleton reorganization and $\mathrm{Ca}^{2+}$ signaling in $\mathrm{T}$ cells Immunol Rev 232: 319-333, 2009.

14. Hall A: Rho family GTPases. Biochem Soc Trans 40: 1378-1382, 2012.

15. Schofield AV and Bernard O: Rho-associated coiled-coil kinase (ROCK) signaling and disease. Crit Rev Biochem Mol Biol 48: 301-316, 2013.

16. Zhou X, Wei M and Wang W: MicroRNA-340 suppresses osteosarcoma tumor growth and metastasis by directly targeting ROCK1. Biochem Biophys Res Commun 437: 653-658, 2013.

17. Bu Q, Tang HM, Tan J, Hu X and Wang DW: Expression of RhoC and ROCK-1 and their effects on MAPK and Akt proteins in prostate carcinoma. Zhonghua Zhong Liu Za Zhi 33: 202-206, 2011 (In Chinese).

18. Majid S, Dar AA, Saini S, et al: MicroRNA-1280 inhibits invasion and metastasis by targeting ROCK1 in bladder cancer. PLoS One 7: e46743, 2012.

19. Lewis BP, Burge CB and Bartel DP: Conserved seed pairing, often flanked by adenosines, indicates that thousands of human genes are microRNA targets. Cell 120: 15-20, 2005.
20. Oellers P, Schroer U, Senner V, Paulus W and Thanos S: ROCKs are expressed in brain tumors and are required for glioma-cell migration on myelinated axons. Glia 57: 499-509, 2009.

21. Inaba N, Ishizawa S, Kimura M, et al: Effect of inhibition of the ROCK isoform on RT2 malignant glioma cells. Anticancer Res 30: 3509-3514, 2010.

22. Hadler-Olsen E, Winberg JO and Uhlin-Hansen L: Matrix metalloproteinases in cancer: their value as diagnostic and prognostic markers and therapeutic targets. Tumour Biol 34: 2041-2051, 2013.

23. Villadsen SB, Bramsen JB, Ostenfeld MS, et al: The miR-143/-145 cluster regulates plasminogen activator inhibitor-1 in bladder cancer. Br J Cancer 106: 366-374, 2012.

24. Chiyomaru T, Tatarano S, Kawakami K, et al: SWAP70, actin-binding protein, function as an oncogene targeting tumorsuppressive miR-145 in prostate cancer. Prostate 71: 1559-1567, 2011.

25. Zhang J, Guo H, Zhang H, et al: Putative tumor suppressor miR-145 inhibits colon cancer cell growth by targeting oncogene Friend leukemia virus integration 1 gene. Cancer 117: 86-95, 2011.

26. Lee HK, Bier A, Cazacu S, et al: MicroRNA-145 is downregulated in glial tumors and regulates glioma cell migration by targeting connective tissue growth factor. PLoS One 8: e54652, 2013.

27. Morgan-Fisher M, Wewer UM and Yoneda A: Regulation of ROCK activity in cancer. J Histochem Cytochem 61: 185-198, 2013.

28. Wen S, Shang Z, Zhu S, Chang C and Niu Y: Androgen receptor enhances entosis, a non-apoptotic cell death, through modulation of Rho/ROCK pathway in prostate cancer cells. Prostate 73: 1306-1315, 2013

29. Wang J, Sun L, Yang M, et al: DEK depletion negatively regulates Rho/ROCK/MLC pathway in non-small cell lung cancer. J Histochem Cytochem 61: 510-521, 2013.

30. Tang AT, Campbell WB and Nithipatikom K: ROCK1 feedback regulation of the upstream small GTPase RhoA. Cell Signal 24: 1375-1380, 2012

31. Imamura F, Mukai M, Ayaki M and Akedo H: Y-27632, an inhibitor of rho-associated protein kinase, suppresses tumor cell invasion via regulation of focal adhesion and focal adhesion kinase. Jpn J Cancer Res 91: 811-816, 2000. 